Research Paper

\title{
Chronic Periodontal Disease increases risk for Prostate Cancer in Elderly individuals in South Korea: a Retrospective Nationwide Population-based Cohort Study
}

\author{
Do-hyung Kim, Seong-Nyum Jeong and Jae-Hong Lee ${ }^{\bowtie}$ \\ Department of Periodontology, Daejeon Dental Hospital, Institute of Wonkwang Dental Research, Wonkwang University College of Dentistry, Daejeon, Korea. \\ $\square$ Corresponding author: Jae-Hong Lee, PhD, Department of Periodontology, Daejeon Dental Hospital, Wonkwang University College of Dentistry, 77, \\ Dunsan-ro, Seo-gu, Daejeon 35233, Korea, E-mail: ljaehong@gmail.com, Tel.: +82-42-3661114, Fax: +82-42-3661115. \\ (c) The author(s). This is an open access article distributed under the terms of the Creative Commons Attribution License (https://creativecommons.org/licenses/by/4.0/). \\ See http://ivyspring.com/terms for full terms and conditions.
}

Received: 2020.02.26; Accepted: 2020.05.17; Published: 2020.05.25

\begin{abstract}
Objectives: The association between prostate cancer (PC) and chronic periodontal disease (PD) has been evaluated in previous studies, but results have been inconsistent. This study aimed to determine whether the presence of chronic PD in old age increases the risk of PC using data in the large-scale elderly cohort.

Materials and Methods: This nationwide population-based cohort study examined data of 121,240 South Korean individuals aged $\geq 60$ years from the National Health Insurance Service-Elderly Cohort database who completed a national program between 2002 and 2015 . For a maximum 10 years' observation period, patients with incident PC with chronic PD compared with those without chronic PD were retrospectively tracked, and Cox proportional hazard ratios and $95 \%$ confidence intervals $(\mathrm{Cls})$ were calculated, adjusted for potential confounding factors, including age, household income, insurance status, Charlson Comorbidity Index, hypertension, diabetes mellitus, cerebral infarction, angina pectoris, myocardial infarction, prostatitis, smoking status, daily smoking, alcohol intake habits, one-time alcohol intake, and regular exercise.

Results: The overall incidence of PC with chronic PD in 10 years was $3.0 \%(n=2,063)$. In the multivariate Cox analysis with adjustment for confounding factors, chronic PD was associated with a $24 \%$ higher risk of $\mathrm{PC}(95 \% \mathrm{Cl}=1.16-1.32, P<0.001)$.

Conclusion: Our results suggest that chronic PD is significantly and positively associated with PC. Larger and better-controlled studies are needed to strengthen this evidence of association and explain the underlying biological mechanisms.
\end{abstract}

Key words: Cohort studies; periodontal disease; periodontitis; prostate cancer, risk factors

\section{Introduction}

Prostate cancer (PC) is one of the most common age-related malignancies in men worldwide and accounts for $20 \%$ of new male cancers in the United States in 2019 [1]. The rate of deaths from PC is decreasing annually but accounts for $9.8 \%$ of deaths from male cancer since 2018 and PC is still a common and significant cause of morbidity and mortality in elderly men [1,2]. In contrast, in 2016, PC was the fourth most common cancer in South Korea after stomach, lung, and colon cancers, with a total of 11,800 cases, accounting for $9.8 \%$ of all male cancers [3]. Especially, recently in South Korea, PC has increased incidence by a greater margin than any other male cancer [3].

Although PC is an extremely popular cancer in elderly men, various factors are intricately 
intertwined with each other, so the primary cause is still controversial $[4,5]$. Congenital factors, such as genetic abnormalities, are closely related to PC, but acquired environments, such as old age and obesity, are also considered important risk factors [6]. Additionally, chronic inflammation is also known to play a decisive role in the initiation, promotion, malignant conversion, invasion, and metastasis of cancer, and the evidence that chronic inflammation is the cause of PC has been reported by epidemiological or pathological studies [7-10].

Chronic periodontal disease (PD) is a representative and typical bacterial and inflammatory disease developing within the oral cavity that results in alveolar bone loss and subsequent loss of teeth for a relatively long period [11,12]. Several risk factors might be responsible for chronic PD, especially smoking, obesity, and diabetes mellitus, which are also major risk factors or risk indicators for chronic PC [13].

Recently, several studies have carefully reported that PC and chronic PD are closely related and correlated with underlying chronic inflammatory processes [14-16]. It is clear that the risk of PC and chronic PD increases with age, and it is considered that patients with chronic PD are more likely to develop PC, but studies are limited. Therefore, this study aimed to determine whether the presence of chronic PD in old age increases the risk of PC using the large-scale elderly cohort database in South Korea.

\section{Materials and Methods}

\section{Source of data}

The study analyses used the National Health Insurance Service-Elderly Cohort (NHIS-EC) database in South Korea, which was established by the NHIS (NHIS sharing service, https://nhiss. nhis.or.kr) to assess the risk and prognostic factors of geriatric disease. In this database, $10 \%$ of elderly participants, who were selected from approximately 5.5 million of elderly individuals aged $\geq 60$ years who are eligible for national health insurance and medical benefits, are included. Data from 2002 to 2015 (14 years), including sociodemographic information, records of medical history and health examination, and information on nursing institutions and long-term care services, were entered in a retrospective cohort form that does not contain personally identifiable or sensitive medical information.

This study conformed to the Strengthening the Reporting of Observational Studies in Epidemiology (STROBE) guidelines for reporting observational cohort studies (www.strobe-statement.org) and was approved by the Institutional Review Board of Daejeon Dental Hospital, Wonkwang University (approval no. W2003/001-001). The requirement for written informed consent was waived by the NHIS (REQ0000034029).

\section{Study population}

We selected the elderly population from those who were included in the NHIS-EC database between January 2002 and December $2005(n=558,147)$. We identified eligible participants $(n=121,240)$ after excluding (1) female $(n=327,565)$, (2) those who had missing general and oral health examinations $(n=$ $109,918)$, and (3) those who had a previous history of cancer or new cancer diagnosis or death between 2002 and $2005(n=322)$. Then, included participants were classified into a group with chronic PD $(n=60,772)$ and group without chronic PD $(n=60,468)$ and followed up for PC diagnosed until December 31, 2015 (Figure 1).

\section{Definitions}

The chronic PD diagnosis was based on the K052-K056 codes of the Korean Classification of Disease 7 th revision (KCD-7), corresponding to codes of the International Classification of Disease 10th revision (ICD-10), according to criteria of the Centers for Disease Control and Prevention/American Academy of Periodontology $[17,18]$. During the oral health examination, chronic PD was assessed and scored based on measurements of clinical periodontal parameters, including bleeding on probing, probing depth, clinical attachment level, and periodontal tooth loss [19]. Moreover, to confirm chronic PD, the participants were limited to those diagnosed with chronic PD at least twice during the oral health examination between 2002 and 2005. The primary endpoint was the newly diagnosed PC in 10 years from 2006 to 2015, which was defined using the KCD-7 code C61, corresponding to ICD-10 code C61, by medical oncologists or urologists.

\section{Covariates}

Sociodemographic information, general and oral health examinations, and self-reported questionnaires were used to collect data at the time of enrollment for the following potential variables regarded as risk factors or indicators for PC and included as covariates in the univariable and multivariable analyses: age (two groups: those aged 60-69 years and those aged $\geq$ 70 years), household income (five groups: those divided into five quintiles based on the insurance fee imposed on each household, with the Medical Aid Program [MAP] group classified into the first quintile), insurance status (three groups: those in the MAP and those in the NHIS groups [self-employed 
and employees]), Charlson Comorbidity Index (CCI, three groups: those divided into three scores and a brief description of the terms related to CCI is provided in Supplementary Material 1), hypertension (KCD-7 codes I10 and I15, corresponding to ICD-10 codes I10 and I15), diabetes mellitus (KCD-7 codes E10-E14, corresponding to ICD-10 codes E10-E14), cerebral infarction (KCD-7 codes I63-I66, corresponding to ICD-10 codes I63-I66), angina pectoris (KCD-7 codes I20, corresponding to ICD-10 codes I20), myocardial infarction (KCD-7 codes I21 and I22, corresponding to ICD-10 codes I21 and I22), prostatitis (KCD-7 code N41, corresponding to ICD-10 code N41), smoking status and number of cigarettes per day, alcohol intake habit and amount of alcohol intake at a time, and regular exercise per week.

\section{Statistical analysis}

Categorical data were shown as frequencies and percentages. Cohort-related variables, including sociodemographic factors (age, household income, and insurance status), comorbidities (CCI, hypertension, diabetes mellitus, cerebral infarction, angina pectoris, myocardial infarction, and prostatitis), self-reported questionnaire (smoking status, daily smoking, alcohol intake habits, one-time alcohol intake, and regular exercise), were analyzed using the Pearson chi-square test. Hazard ratios (HRs) with 95\% confidence intervals (CIs) were calculated via statistical analysis using Cox proportional-hazards regression models after adjusting for sociodemographic factors, comorbidities, and selfreported questionnaire outcomes. The cumulative incidence of PC with and without chronic PD was estimated using the Kaplan-Meier method and compared using the log-rank test. All statistical analyses were conducted using the Statistical Analysis System software version 9.4 (SAS Institute, Cary, NC, USA) and $R$ version 3.5.3 (The $R$ Foundation for Statistical Computing, Vienna, Austria). Statistical significance was considered at two-sided $P$-value $<0.05$.

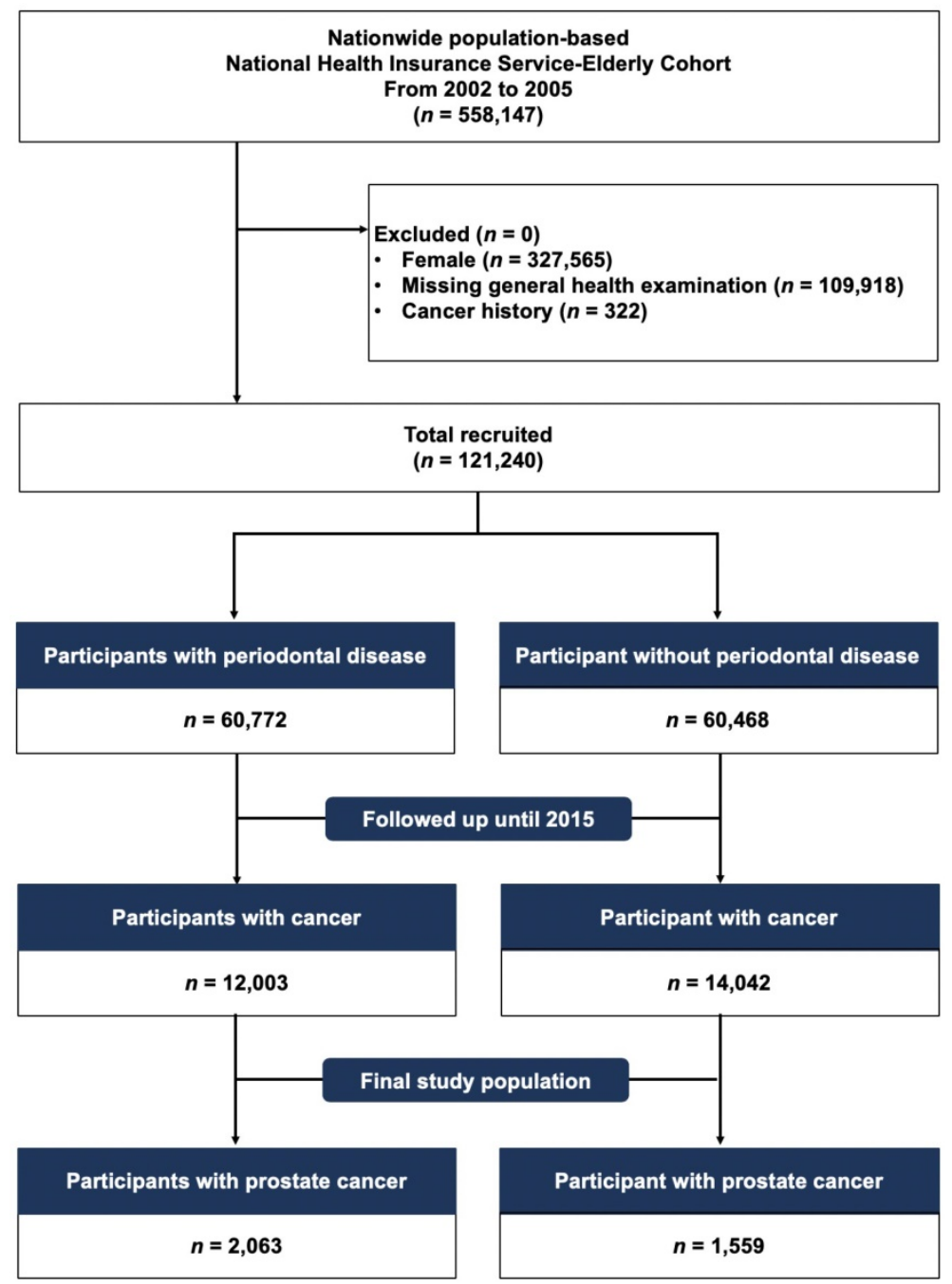

Figure 1. CONSORT flowchart showing the study cohort enrollment process. 
Table 1. Baseline characteristics of the study population included in the National Health Insurance Service-Elderly Cohort according to the presence and absence of chronic periodontal disease (PD)

\begin{tabular}{|c|c|c|c|c|c|}
\hline \multirow{2}{*}{$\begin{array}{l}\text { Variables } \\
\text { Total }\end{array}$} & \multicolumn{2}{|c|}{$\begin{array}{l}\text { Participants with } \\
\text { chronic PD }(n, \%)\end{array}$} & \multicolumn{2}{|c|}{$\begin{array}{l}\text { Participants without } \\
\text { chronic PD }(n, \%)\end{array}$} & \multirow[t]{2}{*}{$P a$} \\
\hline & 60,772 & $(100.0)$ & 60,468 & $(100.0)$ & \\
\hline \multicolumn{6}{|l|}{ Sociodemographics } \\
\hline \multicolumn{6}{|l|}{ Age group (years) } \\
\hline $60-69$ & 49,822 & $(82.0)$ & 42,001 & $(69.5)$ & \multirow[t]{2}{*}{$<0.001$} \\
\hline$\geq 70$ & 10,950 & $(18.0)$ & 18,467 & $(30.5)$ & \\
\hline \multicolumn{6}{|l|}{ Household income } \\
\hline First quintile & 9,550 & $(15.7)$ & 11,063 & $(18.3)$ & \multirow[t]{5}{*}{$<0.001$} \\
\hline Second quintile & 8,052 & $(13.2)$ & 9,949 & $(16.5)$ & \\
\hline Third quintile & 9,799 & $(16.1)$ & 10,284 & $(17.0)$ & \\
\hline Fourth quintile & 13,785 & $(22.7)$ & 13,288 & $(22.0)$ & \\
\hline Fifth quintile & 19,586 & $(32.2)$ & 15,884 & $(26.3)$ & \\
\hline \multicolumn{6}{|l|}{ Insurance status } \\
\hline MAP & 198 & $(0.3)$ & 351 & $(0.6)$ & \multirow[t]{3}{*}{$<0.001$} \\
\hline NHIS (self-employed) & 35,658 & $(58.7)$ & 32,797 & $(54.2)$ & \\
\hline NHIS (employees) & 24,916 & $(41.0)$ & 27,320 & $(45.2)$ & \\
\hline \multicolumn{6}{|l|}{ Comorbidity } \\
\hline \multicolumn{6}{|l|}{$C C I$} \\
\hline 0 & 31,378 & $(51.6)$ & 32,811 & $(54.3)$ & \multirow[t]{3}{*}{$<0.001$} \\
\hline 1 & 15,484 & $(25.5)$ & 14,454 & $(23.9)$ & \\
\hline$\geq 2$ & 13,910 & $(22.9)$ & 13,203 & $(21.8)$ & \\
\hline \multicolumn{6}{|l|}{ Hypertension } \\
\hline Yes & 40,660 & $(66.9)$ & 37,308 & $(61.7)$ & \multirow[t]{2}{*}{$<0.001$} \\
\hline No & 20,112 & $(33.1)$ & 23,160 & $(38.3)$ & \\
\hline \multicolumn{6}{|l|}{ Diabetes mellitus } \\
\hline Yes & 22,761 & $(37.5)$ & 18,472 & $(30.5)$ & \multirow[t]{2}{*}{$<0.001$} \\
\hline No & 38,011 & $(62.5)$ & 41,996 & $(69.5)$ & \\
\hline Cerebral infarction & & & & & \\
\hline Yes & 12,879 & $(21.2)$ & 13,216 & $(21.9)$ & $<0.001$ \\
\hline No & 47,893 & $(78.8)$ & 47,252 & $(78.1)$ & \\
\hline Angina pectoris & & & & & \\
\hline Yes & 2,553 & $(4.2)$ & 2,439 & $(4.0)$ & 0.004 \\
\hline No & 58,219 & $(95.8)$ & 58,029 & $(96.0)$ & \\
\hline Myocardial infarction & & & & & \\
\hline Yes & 12,321 & $(20.3)$ & 9,296 & $(15.4)$ & $<0.001$ \\
\hline No & 48,451 & $(79.7)$ & 51,172 & $(84.6)$ & \\
\hline Prostatitis & & & & & \\
\hline Yes & 7,144 & $(11.8)$ & 5,157 & $(8.5)$ & $<0.001$ \\
\hline No & 53,628 & $(88.2)$ & 55,311 & $(91.5)$ & \\
\hline $\begin{array}{l}\text { Self-reported } \\
\text { questionnaire }\end{array}$ & & & & & \\
\hline Smoking status & & & & & \\
\hline Yes & 17,907 & $(29.5)$ & 19,557 & $(32.3)$ & $<0.001$ \\
\hline No & 42,865 & $(70.5)$ & 40,911 & $(67.7)$ & \\
\hline Daily smoking & & & & & \\
\hline$<20$ cigarettes & 15,129 & $(84.5)$ & 16,799 & $(85.9)$ & $<0.001$ \\
\hline$\geq 20$ cigarettes & 2,778 & $(15.5)$ & 2,758 & $(14.1)$ & \\
\hline Alcohol intake habits & & & & & \\
\hline No drinking & 30,304 & $(49.9)$ & 32,071 & $(53.0)$ & $<0.001$ \\
\hline 1-4 times/week & 23,588 & $(38.8)$ & 20,087 & $(33.2)$ & \\
\hline Almost daily & 6,880 & $(11.3)$ & 8,310 & $(13.7)$ & \\
\hline One-time alcohol intake & & & & & \\
\hline$<1$ bottle of beer $(360 \mathrm{~mL})$ & 27,095 & $(88.9)$ & 25,254 & $(88.9)$ & 0.992 \\
\hline$\geq 1$ bottle of beer $(360 \mathrm{~mL})$ & 3,373 & $(11.1)$ & 3,143 & $(11.1)$ & \\
\hline Regular exercise & & & & & \\
\hline No & 32,457 & $(53.4)$ & 39,529 & $(65.4)$ & $<0.001$ \\
\hline 1-4 times/week & 17,623 & $(29.0)$ & 12,431 & $(20.6)$ & \\
\hline$\geq 5$ times/week & 10,692 & $(17.6)$ & 8,508 & $(14.1)$ & \\
\hline
\end{tabular}

MAP, Medical Aid Program; NHIS, National Health Insurance Service; CCI, Charlson Comorbidity Index.

a $P$-values were obtained using the Pearson chi-square test.

bivided into five quintiles based on the insurance fee imposed on each household, with the MAP group classified into the first quintile.

\section{Results}

\section{Baseline characteristics of the study population}

Table 1 shows the baseline characteristics of the study population according to the presence and absence of chronic PD. Participants with chronic PD were more likely to have comorbidities, such as hypertension $(66.9 \%)$, diabetes mellitus $(37.5 \%)$, angina pectoris $(4.2 \%)$, myocardial infarction $(20.3 \%)$, and prostatitis $(11.8 \%)$, and less likely to smoke (29.5\%) and perform regular physical activity (53.4\%) compared with those without chronic PD.

\section{Cumulative incidence of PC}

Table 2 shows the cumulative incidence of PC during the follow-up period after diagnosis of chronic PD. During the study period, of participants with chronic PD $(n=2,063), 1,487 \quad(72.1 \%)$ had hypertension, $827(40.1 \%)$ had diabetes mellitus, 490 $(23.8 \%)$ had cerebral infarction, $83(4.0 \%)$ had angina pectoris, $560(27.1 \%)$ had myocardial infarction, and $465(22.5 \%)$ had prostatitis. In contrast, in those without chronic PD $(n=1,559), 1,033(66.3 \%)$ had hypertension, $553(35.5 \%)$ had diabetes mellitus, 331 $(21.2 \%)$ had cerebral infarction, 66 (4.2\%) had angina pectoris, $303(19.4 \%)$ had myocardial infarction, and 307 (19.7\%) had prostatitis.

\section{Risk of PC}

Table 3 shows the univariable and multivariable analyses, with adjustment for sociodemographic factors, comorbidities, smoking, alcohol consumption, and physical activity. The results of the multivariable analysis indicated that the incidence of PC was significantly positively correlated with older age $(\geq 70$ years, adjusted HR 1.37, 95\% CI 1.27-1.48, $P<0.001$ ), higher household incomes (fourth and fifth quintiles, adjusted HR 1.21, 95\% CI 1.08-1.35, $P<0.001$ and adjusted HR 1.28, 95\% CI 1.15-1.42, $P<0.001$, respectively), higher CCI score ( $>2$, adjusted HR 1.19, 95\% CI 1.10-1.29, $P<0.001$ ), hypertension (adjusted HR 1.13, 95\% CI 1.05-1.22, $P=0.001$ ), diabetes mellitus (adjusted HR 1.08, 95\% CI 1.00-1.16, $P=$ 0.041 ), angina pectoris (adjusted HR 1.24, 95\% CI 1.14-1.34, $P<0.001$ ), prostatitis (adjusted HR 1.92, 95\% CI 1.77-2.08, $P<0.001$ ), and current smoking (adjusted HR 1.24, 95\% CI 1.14-1.33, $P<0.001$ ).

\section{Association of PD with incidence of PC}

In the initial study population, 3,622 participants were diagnosed with PC during the follow-up period, and a higher risk of PC incidence was observed in the group with chronic $\mathrm{PD}$ than in the group without chronic PD (unadjusted HR 1.31, 95\% CI 1.23-1.40, $P<$ 
0.001; adjusted HR 1.24, 95\% CI 1.16-1.32, $P<0.001$ ). The Kaplan-Meier curve also showed the risk of incident PC in the groups with and without chronic PD (Figure 2).

\section{Discussion}

In this study, the proportion of PCs among cancers in elderly men was $13.9 \%$, which is similar to that in the World Health Organization GLOBOCAN database that reported $15 \%$ of PCs among all male cancers, and these results are also highly consistent with the increase in PC incidence and prevalence at older ages.[6] Our study, which was conducted on 121,240 men aged $\geq 60$ years, showed that the incidence of PC with chronic PD in 10 years was $3.0 \%$, and adjusted HR for developing a new PC was 1.24 (95\% CI 1.16-1.32, $P<0.001$ ) [15].

Despite the growing interest in oral health and improvement of oral hygiene and dental services, the incidence and prevalence of chronic PD have been consistently increasing as society ages [20]. In 2018, PD was the second most common disease in the outpatient hospital care in South Korea, and about $30.1 \%$ (15.6 million individuals) of its current population has already received treatment. Previously, chronic PD was defined as a localized and chronic inflammatory response caused by specific anaerobic bacteria in the periodontal pocket, such as Porphyromonas gingivalis, Tannerella forsythia, Treponema denticola, and Aggregatibacter actinomycetemcomitans. However, recently, clinical and pathological evidence that chronic PD is closely related to chronic and progressive systemic inflammatory diseases, including cardiovascular disease, diabetes mellitus, rheumatoid arthritis, and osteoporosis, has steadily emerged [21-23].

Recent studies have reported that chronic PD affects the development and/or progression of several types of cancer, including oral and oropharyngeal cancer $[16,24]$. Especially with the exception of orofacial cancer, after adjusting for known risk factors, such as age, sex, and smoking, chronic PD was statistically significantly associated with an increased risk of pancreatic cancer (Michaud et al., HR 1.64, 95\% CI 1.19-2.26; Chang et al., HR 1.55, 95\% CI 1.02-2.33; Maisonneuve et al., HR 1.74, 95\% CI 1.41-2.15) [25-27].

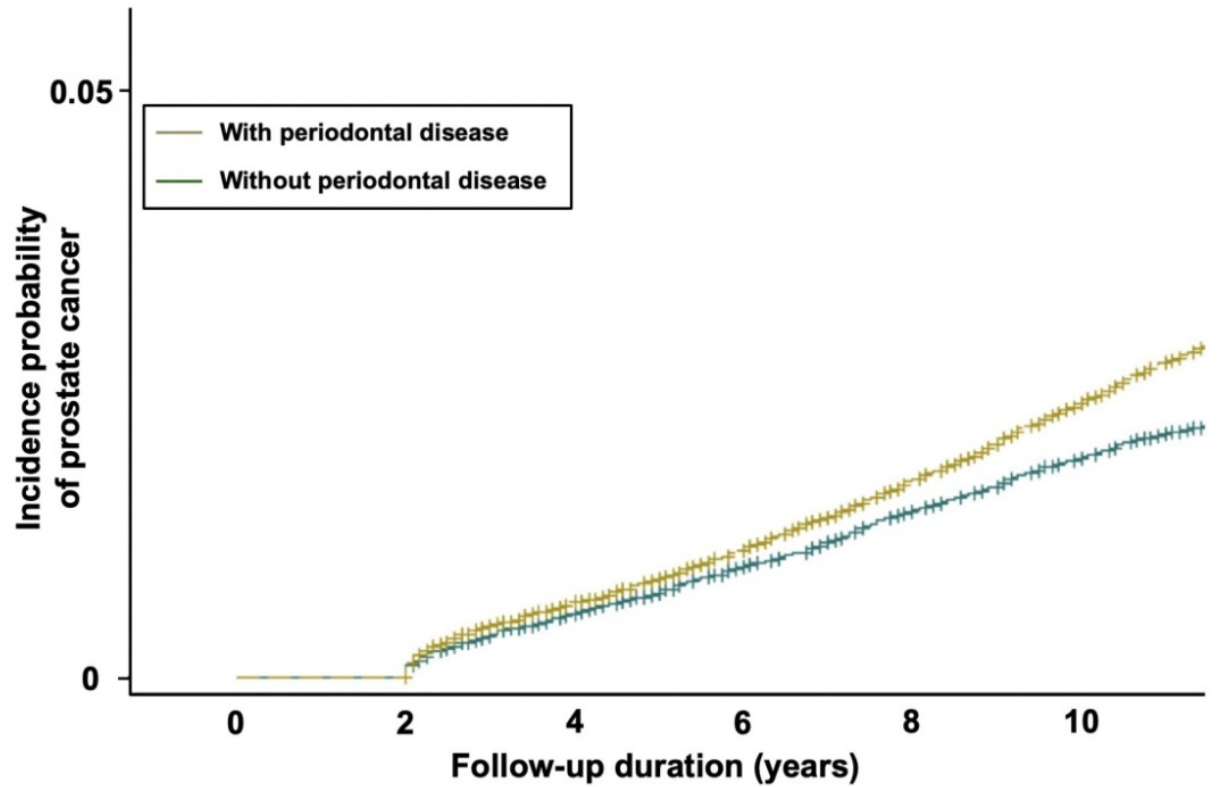

Number at risk

$\begin{array}{rllllll}\text { With periodontal disease } & 60,772 & 60,760 & 60,279 & 59,954 & 59,555 & 58,709 \\ \text { Without periodontal disease } & 60,468 & 60,461 & 60,069 & 59,790 & 59,476 & 58,909\end{array}$

The risk of incident prostate cancer

\begin{tabular}{|c|c|c|c|c|c|c|}
\hline \multirow[b]{2}{*}{ Variables } & \multicolumn{3}{|c|}{ Univariable analysis } & \multicolumn{3}{|c|}{ Multivariable analysis } \\
\hline & HR & $95 \% \mathrm{Cl}$ & $P$ & HR & $95 \% \mathrm{Cl}$ & $P$ \\
\hline Periodontal disease (reference: no) & 1.31 & $1.23-1.40$ & $<0.001$ & 1.24 & $1.16-1.32$ & $<0.001$ \\
\hline
\end{tabular}

Figure 2. Kaplan-Meier analysis showing the cumulative incidence rates of prostate cancer with and without chronic periodontal disease during the follow-up periods. The risk of incident prostate cancer with chronic periodontal disease was higher and significantly different from that of prostate cancer without chronic periodontal disease $(P<0.001)$. 
Table 2. Cumulative incidence of prostate cancer in participants with and without chronic PD

\begin{tabular}{|c|c|c|c|c|c|}
\hline \multirow{3}{*}{$\begin{array}{l}\text { Variables } \\
\\
\text { Total }\end{array}$} & \multicolumn{4}{|c|}{$\begin{array}{l}\text { Cumulative incidence of prostate cancer at } \\
10 \text { years }\end{array}$} & \multirow[t]{3}{*}{$\mathrm{Pa}$} \\
\hline & \multicolumn{2}{|c|}{$\begin{array}{l}\text { Participants with } \\
\text { chronic PD }(n, \%)\end{array}$} & \multicolumn{2}{|c|}{$\begin{array}{l}\text { Participants without } \\
\text { chronic PD }(n, \%)\end{array}$} & \\
\hline & 2,063 & $(100.0)$ & 1,559 & $(100.0)$ & \\
\hline \multicolumn{6}{|l|}{ Sociodemographics } \\
\hline \multicolumn{6}{|l|}{ Age group (years) } \\
\hline $60-69$ & 1,533 & $(74.3)$ & 978 & $(62.7)$ & $<0.001$ \\
\hline$\geq 70$ & 530 & $(25.7)$ & 581 & $(37.3)$ & \\
\hline \multicolumn{6}{|l|}{ Household income } \\
\hline First quintile & 236 & $(11.4)$ & 284 & $(18.2)$ & $<0.001$ \\
\hline Second quintile & 227 & $(11.0)$ & 192 & (12.3) & \\
\hline Third quintile & 303 & $(14.7)$ & 226 & $(14.5)$ & \\
\hline Fourth quintile & 489 & $(23.7)$ & 360 & $(23.1)$ & \\
\hline Fifth quintile & 808 & $(39.2)$ & 497 & $(31.9)$ & \\
\hline \multicolumn{6}{|l|}{ Insurance status } \\
\hline MAP & 7 & $(0.3)$ & 13 & $(0.8)$ & 0.021 \\
\hline NHIS (self-employed) & 1,224 & $(59.3)$ & 871 & $(55.9)$ & \\
\hline NHIS (employees) & 832 & $(40.3)$ & 675 & $(43.3)$ & \\
\hline \multicolumn{6}{|l|}{ Comorbidity } \\
\hline \multicolumn{6}{|l|}{$C C I$} \\
\hline 0 & 907 & $(44.0)$ & 775 & $(49.7)$ & 0.001 \\
\hline 1 & 506 & $(24.5)$ & 364 & $(23.3)$ & \\
\hline$\geq 2$ & 650 & $(31.5)$ & 420 & $(26.9)$ & \\
\hline \multicolumn{6}{|l|}{ Hypertension } \\
\hline Yes & 1,487 & $(72.1)$ & 1,033 & $(66.3)$ & $<0.001$ \\
\hline No & 576 & $(27.9)$ & 526 & (33.7) & \\
\hline \multicolumn{6}{|l|}{ Diabetes mellitus } \\
\hline Yes & 827 & $(40.1)$ & 553 & $(35.5)$ & 0.004 \\
\hline No & 1,236 & $(59.9)$ & 1,006 & $(64.5)$ & \\
\hline \multicolumn{6}{|l|}{ Cerebral infarction } \\
\hline Yes & 490 & $(23.8)$ & 331 & $(21.2)$ & 0.072 \\
\hline No & 1,573 & $(76.2)$ & 1,228 & (78.8) & \\
\hline \multicolumn{6}{|l|}{ Angina pectoris } \\
\hline Yes & 83 & $(4.0)$ & 66 & $(4.2)$ & 0.752 \\
\hline No & 1,980 & $(96.0)$ & 1,493 & $(95.8)$ & \\
\hline \multicolumn{6}{|l|}{ Myocardial infarction } \\
\hline Yes & 560 & $(27.1)$ & 303 & $(19.4)$ & $<0.001$ \\
\hline No & 1,503 & $(72.9)$ & 1,256 & $(80.6)$ & \\
\hline \multicolumn{6}{|l|}{ Prostatitis } \\
\hline Yes & 465 & $(22.5)$ & 307 & $(19.7)$ & 0.038 \\
\hline No & 1,598 & $(77.5)$ & 1,252 & $(80.3)$ & \\
\hline Self-reported questionnair & & & & & \\
\hline Smoking status & & & & & \\
\hline Yes & 1,061 & $(51.4)$ & 632 & $(40.5)$ & $<0.001$ \\
\hline No & 1,002 & $(48.6)$ & 927 & $(59.5)$ & \\
\hline Daily smoking & & & & & \\
\hline$<20$ cigarettes & 871 & $(82.1)$ & 554 & $(87.7)$ & 0.023 \\
\hline$\geq 20$ cigarettes & 190 & $(17.9)$ & 78 & $(12.3)$ & \\
\hline Alcohol intake habits & & & & & \\
\hline No drinking & 1,137 & $(55.1)$ & 914 & $(58.6)$ & $<0.001$ \\
\hline 1-4 times/week & 753 & $(36.5)$ & 478 & $(30.7)$ & \\
\hline Almost daily & 173 & $(8.4)$ & 167 & $(10.7)$ & \\
\hline One-time alcohol intake & & & & & \\
\hline$<1$ bottle of beer $(360 \mathrm{~mL})$ & 822 & $(88.8)$ & 580 & $(89.9)$ & 0.468 \\
\hline$\geq 1$ bottle of beer $(360 \mathrm{~mL})$ & 104 & $(11.2)$ & 65 & $(10.1)$ & \\
\hline Regular exercise & & & & & \\
\hline No & 1,587 & $(76.9)$ & 1,154 & $(74.0)$ & $<0.001$ \\
\hline 1-4 times/week & 377 & $(18.3)$ & 181 & $(11.6)$ & \\
\hline$\geq 5$ times/week & 99 & $(4.8)$ & 224 & (14.4) & \\
\hline
\end{tabular}

a $P$-values were obtained using the Pearson chi-square test.

In contrast, few studies have reported positive associations between PC and chronic PD [15,28]. In our previous study on 187,934 adult men aged $\geq 40$ using data from a South Korean nationwide population based on the NHIS-Health Examinee Cohort, the incidence of PC with chronic PD in 12 years was $0.5 \%$, and adjusted HR for developing a new PC within 12 years was 1.14 (95\% CI 1.01-1.31, $P$ $=0.042$ ) [15]. Another epidemiological cohort study using the Turkish National Cancer Registry database also found that men aged $\geq 35$ years with PD were at high risk for developing PC in a follow-up of 12 years (standardized incidence rates 3.75, 95\% CI 0.95-10.21, $P=0.014)[28]$.

Table 3. Univariable and multivariable Cox proportional hazards regression analyses for factors potentially affecting the development of prostate cancer

\begin{tabular}{|c|c|c|c|c|c|c|}
\hline & \multicolumn{3}{|c|}{ Univariable analysis } & \multicolumn{3}{|c|}{ Multivariable analysis } \\
\hline Variables & HR & $95 \% \mathrm{CI}$ & $P$ & HR & $95 \% \mathrm{CI}$ & $P$ \\
\hline \multicolumn{7}{|l|}{ Sociodemographics } \\
\hline \multicolumn{7}{|l|}{$\begin{array}{l}\text { Age group } \\
\text { (reference: } 60-69 \text { years) }\end{array}$} \\
\hline$\geq 70$ & 1.32 & $1.23-1.41$ & $<0.001$ & 1.37 & $1.27-1.48$ & $<0.001$ \\
\hline \multicolumn{7}{|l|}{$\begin{array}{l}\text { Household income } \\
\text { (reference: first quintile) }\end{array}$} \\
\hline Second quintile & 0.95 & $0.83-1.08$ & 0.402 & 0.99 & $0.87-1.13$ & 0.855 \\
\hline Third quintile & 1.05 & $0.93-1.18$ & 0.468 & 1.06 & $0.94-1.20$ & 0.362 \\
\hline Fourth quintile & 1.23 & $1.10-1.37$ & $<0.001$ & 1.21 & $1.08-1.35$ & $<0.001$ \\
\hline Fifth quintile & 1.40 & $1.27-1.55$ & $<0.001$ & 1.28 & $1.15-1.42$ & $<0.001$ \\
\hline \multicolumn{7}{|l|}{$\begin{array}{l}\text { Insurance status } \\
\text { (reference: } M A P)\end{array}$} \\
\hline NHIS (self-employed) & 0.77 & $0.49-1.19$ & 0.237 & 0.62 & $0.39-0.96$ & 0.034 \\
\hline NHIS (employees) & 0.73 & $0.47-1.14$ & 0.168 & 0.62 & $0.39-0.97$ & 0.036 \\
\hline \multicolumn{7}{|l|}{ Comorbidity } \\
\hline \multicolumn{7}{|l|}{ CCI (reference: score 0) } \\
\hline 1 & 1.09 & $1.00-1.18$ & 0.051 & 1.03 & $0.95-1.12$ & 0.441 \\
\hline$\geq 2$ & 1.30 & $1.20-1.40$ & $<0.001$ & 1.19 & $1.10-1.29$ & $<0.001$ \\
\hline Hypertension (reference: no) & 1.23 & $1.14-1.32$ & $<0.001$ & 1.13 & $1.05-1.22$ & 0.001 \\
\hline $\begin{array}{l}\text { Diabetes mellitus } \\
\text { (reference: no) }\end{array}$ & 1.15 & $1.08-1.23$ & $<0.001$ & 1.08 & $1.00-1.16$ & 0.041 \\
\hline $\begin{array}{l}\text { Cerebral infarction } \\
\text { (reference: no) }\end{array}$ & 1.03 & $0.95-1.11$ & 0.444 & 0.94 & $0.87-1.02$ & 0.114 \\
\hline $\begin{array}{l}\text { Angina pectoris } \\
\text { (reference: no) }\end{array}$ & 1.35 & $1.25-1.46$ & $<0.001$ & 1.24 & $1.14-1.34$ & $<0.001$ \\
\hline $\begin{array}{l}\text { Myocardial infarction } \\
\text { (reference: no) }\end{array}$ & 0.98 & $0.83-1.15$ & 0.787 & 0.80 & $0.68-0.95$ & 0.010 \\
\hline Prostatitis (reference: no) & 2.07 & $1.91-2.25$ & $<0.001$ & 1.92 & $1.77-2.08$ & $<0.001$ \\
\hline \multicolumn{7}{|l|}{ Self-reported questionnaire } \\
\hline $\begin{array}{l}\text { Smoking status } \\
\text { (reference: no) }\end{array}$ & 1.32 & $1.22-1.42$ & $<0.001$ & 1.24 & $1.14-1.33$ & $<0.001$ \\
\hline $\begin{array}{l}\text { Daily smoking } \\
\text { (reference: }<20 \text { cigarettes) }\end{array}$ & 1.18 & $1.08-1.29$ & $<0.001$ & 1.08 & $0.98-1.18$ & 0.109 \\
\hline \multicolumn{7}{|l|}{$\begin{array}{l}\text { Alcohol intake habits } \\
\text { (reference: no drinking) }\end{array}$} \\
\hline 1-4 times/week & 0.88 & $0.82-0.95$ & $<0.001$ & 0.92 & $0.86-1.00$ & 0.036 \\
\hline Almost daily & 0.70 & $0.63-0.79$ & $<0.001$ & 0.84 & $0.74-0.95$ & 0.004 \\
\hline $\begin{array}{l}\text { One-time alcohol intake } \\
\text { (reference: < } 1 \text { bottle) }\end{array}$ & 0.87 & $0.75-1.02$ & 0.076 & 1.05 & $0.90-1.24$ & 0.522 \\
\hline \multicolumn{7}{|l|}{$\begin{array}{l}\text { Regular exercise } \\
\text { (reference: } n o \text { ) }\end{array}$} \\
\hline 1-4 times/week & 0.76 & $0.70-0.81$ & $<0.001$ & 0.86 & $0.79-0.93$ & $<0.001$ \\
\hline$\geq 5$ times/week & 0.84 & $0.71-1.00$ & 0.053 & 1.08 & $0.90-1.30$ & 0.428 \\
\hline
\end{tabular}

Dysbiosis of the oral microbiota, bacteriainduced immune evasion and dysregulation, modulation of various signaling pathways, and subsequent inhibition of apoptosis and activation of cell proliferation in patients with chronic PD have all 
been proposed as pro-tumorigenic mechanisms [29-31]. Moreover, some genes (particularly CDKN2B) that are significantly and consistently related with cancer are also associated with PD, which suggests shared genetic susceptibility between the two diseases [28].

Activated inflammatory cells, including neutrophils, macrophages, and dendritic cells, secrete pro-inflammatory and pro-growth substances, such as tumor necrosis factor (TNF)-a, cytokines, chemokines, matrix metalloproteases, and pro-angiogenic molecules. These cells also produce reactive oxygen and nitrogen species, which can induce DNA damage in epithelial cells and produce an environment for both initiation and promotion of carcinogenesis at local and distant sites [32-34]. Periodontal pathogens might promote cancer development through invasion of blood vessels, bacteremia, and subclinical infection in distant sites [28].

Among the comorbidities investigated in this study, prostatitis was found to have the closest relationship with PC (crude HR 2.07, 95\% CI 1.912.25, $P<0.001$; adjusted HR 1.92, 95\% CI 1.77-2.08, $P<$ 0.001). Patients with moderate-to-severe prostatitis and chronic PD were found to have higher prostate-specific antigen levels, which are used to track response to androgen deprivation therapy (ADT) for PC [35]. Famili et al. found that men with PC undergoing ADT were more likely to have PD than men not undergoing ADT [36]. Both PD and prostatitis result in cytokine imbalance toward increased pro-inflammatory cytokines, such as interleukine (IL)-6, IL-8, IL-18, TNF- $a$, and C-reactive protein, and decreased anti-inflammatory cytokines [37]. Additionally, major clinical parameters of PD, especially clinical attachment level, were significantly worse in patients with moderate-to-severe prostatitis $[35,37]$. Considering the similarity in the etiopathogenesis of prostatitis and PD, it is possible that there is a pathological link between them; however, further studies are necessary to draw a conclusion.

The present study has several limitations. First, since all patients with cancer are required to register in the National Cancer Registration and Statistics system, the diagnostic accuracy of PC is highly reliable, while it is difficult to guarantee the diagnostic accuracy of chronic PD due to the nature of this study. Therefore, to increase the diagnostic reliability of chronic PD, diagnosis should be made twice using clinical parameters of the oral health examination, and all participants who did not undergo oral health examination were excluded. Second, because the current NHIS-EC database does not provide information on the stage and grade of $\mathrm{PC}$, it is difficult to confirm the association between the progression or severity of PC and chronic PD. Third, due to the study design, it is hard to distinguish whether PD and PC have a causative relationship or they share the same susceptibility. Finally, since this study has been analyzed retrospectively only for those who have undergone general and oral health examinations, selection bias could occur fundamentally.

\section{Conclusion}

Despite the abovementioned limitations, this is the first study that used NHIS-based elderly cohort data from South Korea, and the results demonstrated a positive and significant association between PC and chronic PD. The retrospective nature of this study limits the conclusiveness of our findings. Therefore, large better controlled studies are warranted to strengthen this evidence of association and help elucidate the underlying biological mechanisms.

\section{Supplementary Material}

Supplementary figures and tables. http://www.jcancer.org/v11p4716s1.pdf

\section{Acknowledgements}

The used NHIS-HEALS data (NHIS-2020-2-082) were supplied by the NHIS. The authors declare that they have no potential conflicts of interest with the NHIS or financial disclosures to report. This work was supported by a National Research Foundation of Korea (NRF) grant funded by the Korean government (MSIT) (No. 2019R1A2C1083978) and Wonkwang University in 2020.

\section{Competing Interests}

The authors have declared that no competing interest exists.

\section{References}

1. Siegel RL, Miller KD, Jemal A. Cancer statistics, 2019. CA Cancer J Clin 2019; 69(1):7-34.

2. Mohler JL, Antonarakis ES, Armstrong AJ, D'Amico AV, Davis BJ, Dorff T, et al. Prostate Cancer, Version 2.2019, NCCN Clinical Practice Guidelines in Oncology. J Natl Compr Canc Netw 2019; 17(5):479-505.

3. Jung KW, Won YJ, Kong HJ, Lee ES. Prediction of Cancer Incidence and Mortality in Korea, 2018. Cancer Res Treat 2018; 50(2):317-23.

4. Hsing AW, Chokkalingam AP. Prostate cancer epidemiology. Front Biosci 2006; 11:1388-413.

5. Witte JS. Prostate cancer genomics: towards a new understanding. Nat Rev Genet 2009; 10(2):77-82.

6. Torre LA, Bray F, Siegel RL, Ferlay J, Lortet-Tieulent J, Jemal A. Global cancer statistics, 2012. CA Cancer J Clin 2015; 65(2):87-108.

7. Mantovani A, Allavena P, Sica A, Balkwill F. Cancer-related inflammation. Nature 2008; 454(7203):436-44.

8. Grivennikov SI, Greten FR, Karin M. Immunity, inflammation, and cancer. Cell 2010; 140(6):883-99.

9. DeMarzo AM, Nelson WG, Isaacs WB, Epstein JI. Pathological and molecular aspects of prostate cancer. Lancet 2003; 361(9361):955-64.

10. Gurel B, Lucia MS, Thompson IM, Jr., Goodman PJ, Tangen CM, Kristal AR, et al. Chronic inflammation in benign prostate tissue is associated with high-grade prostate cancer in the placebo arm of the prostate cancer prevention trial. Cancer Epidemiol Biomarkers Prev 2014; 23(5):847-56. 
11. Lee JH, Oh JY, Choi JK, Kim YT, Park YS, Jeong SN, et al. Trends in the incidence of tooth extraction due to periodontal disease: results of a 12-year longitudinal cohort study in South Korea. J Periodontal Implant Sci 2017; 47(5):264-72.

12. Kim YT, Choi JK, Kim DH, Jeong SN, Lee JH. Association between health status and tooth loss in Korean adults: longitudinal results from the National Health Insurance Service-Health Examinee Cohort, 2002-2015. J Periodontal Implant Sci 2019; 49(3):158-70.

13. Gong Z, Neuhouser ML, Goodman PJ, Albanes D, Chi C, Hsing AW, et al. Obesity, diabetes, and risk of prostate cancer: results from the prostate cancer prevention trial. Cancer Epidemiol Biomarkers Prev 2006; 15(10):1977-83.

14. Palapattu GS, Sutcliffe S, Bastian PJ, Platz EA, De Marzo AM, Isaacs WB, et al. Prostate carcinogenesis and inflammation: emerging insights. Carcinogenesis 2005; 26(7):1170-81.

15. Lee JH, Kweon HH, Choi JK, Kim YT, Choi SH. Association between Periodontal disease and Prostate cancer: Results of a 12-year Longitudinal Cohort Study in South Korea. J Cancer 2017; 8(15):2959-65.

16. Fitzpatrick SG, Katz J. The association between periodontal disease and cancer: a review of the literature. J Dent 2010; 38(2):83-95.

17. Armitage GC. Development of a classification system for periodontal diseases and conditions. Ann Periodontol 1999; 4(1):1-6.

18. Page RC, Eke PI. Case definitions for use in population - Based surveillance of periodontitis. J Periodontol 2007; 78(7):1387-99.

19. Kingman A, Albandar JM. Methodological aspects of epidemiological studies of periodontal diseases. Periodontol 2000 2002; 29:11-30.

20. Lee JH, Lee JS, Choi JK, Kweon HI, Kim YT, Choi SH. National dental policies and socio-demographic factors affecting changes in the incidence of periodontal treatments in Korean: A nationwide population-based retrospective cohort study from 2002-2013. BMC Oral Health 2016; 16(1):118.

21. Lee JH, Lee JS, Park JY, Choi JK, Kim DW, Kim YT, et al. Association of Lifestyle-Related Comorbidities With Periodontitis: A Nationwide Cohort Study in Korea. Medicine (Baltimore) 2015; 94(37):e1567.

22. Lee JH, Oh JY, Youk TM, Jeong SN, Kim YT, Choi SH. Association between periodontal disease and non-communicable diseases: A 12-year longitudinal health-examinee cohort study in South Korea. Medicine (Baltimore) 2017; 96(26):e7398.

23. Choi JK, Kim YT, Kweon HI, Park EC, Choi SH, Lee JH. Effect of periodontitis on the development of osteoporosis: results from a nationwide population-based cohort study (2003-2013). BMC Womens Health 2017; 17(1):77.

24. Heikkila P, But A, Sorsa T, Haukka J. Periodontitis and cancer mortality: Register-based cohort study of 68,273 adults in 10-year follow-up. Int J Cancer 2018; 142(11):2244-53.

25. Michaud DS, Joshipura K, Giovannucci E, Fuchs CS. A prospective study of periodontal disease and pancreatic cancer in US male health professionals. J Natl Cancer Inst 2007; 99(2):171-5.

26. Chang JS, Tsai CR, Chen LT, Shan YS. Investigating the Association Between Periodontal Disease and Risk of Pancreatic Cancer. Pancreas 2016; 45(1):134-41.

27. Maisonneuve P, Amar S, Lowenfels AB. Periodontal disease, edentulism, and pancreatic cancer: a meta-analysis. Ann Oncol 2017; 28(5):985-95.

28. Dizdar O, Hayran M, Guven DC, Yilmaz TB, Taheri S, Akman AC, et al. Increased cancer risk in patients with periodontitis. Curr Med Res Opin 2017; 33(12):2195-200.

29. Perera M, Al-Hebshi NN, Speicher DJ, Perera I, Johnson NW. Emerging role of bacteria in oral carcinogenesis: a review with special reference to perio-pathogenic bacteria. J Oral Microbiol 2016; 8:32762.

30. Schmidt J, Jentsch H, Stingu CS, Sack U. General immune status and oral microbiology in patients with different forms of periodontitis and healthy control subjects. PLoS One 2014; 9(10):e109187.

31. Cifcibasi E, Ciblak M, Kiran B, Badur S, Firatli E, Issever H, et al. The role of activated cytotoxic $\mathrm{T}$ cells in etiopathogenesis of periodontal disease: does it harm or does it heal? Sci Rep 2015; 5:9262.

32. Seymour GJ, Ford PJ, Cullinan MP, Leishman S, Yamazaki K. Relationship between periodontal infections and systemic disease. Clin Microbiol Infect 2007; 13 Suppl 4:3-10.

33. Mai X, LaMonte MJ, Hovey KM, Freudenheim JL, Andrews CA, Genco RJ, et al. Periodontal disease severity and cancer risk in postmenopausal women: the Buffalo OsteoPerio Study. Cancer Causes Control 2016; 27(2):217-28.

34. Javed F, Warnakulasuriya S. Is there a relationship between periodontal disease and oral cancer? A systematic review of currently available evidence. Crit Rev Oncol Hematol 2016; 97:197-205.

35. Boyapati R, Swarna C, Devulapalli N, Sanivarapu S, Katuri KK, Kolaparthy L. Unveiling the Link between Prostatitis and Periodontitis. Contemp Clin Dent 2018; 9(4):524-9.

36. Famili P, Cauley JA, Greenspan SL. The effect of androgen deprivation therapy on periodontal disease in men with prostate cancer. J Urol 2007; 177(3):921-4.

37. Joshi N, Bissada NF, Bodner D, Maclennan GT, Narendran S, Jurevic R, et al. Association between periodontal disease and prostate-specific antigen levels in chronic prostatitis patients. J Periodontol 2010; 81(6):864-9. 